\title{
Transcranial Magnetic Stimulation over Sensorimotor Cortex Disrupts Anticipatory Reflex Gain Modulation for Skilled Action
}

\author{
Toshitaka Kimura, ${ }^{1}$ Patrick Haggard, ${ }^{2}$ and Hiroaki Gomi ${ }^{1}$ \\ ${ }^{1}$ NTT Communication Science Laboratories, Nippon Telegraph and Telephone Corporation, Kanagawa 243-0198, Japan, and ${ }^{2}$ Institute of Cognitive \\ Neuroscience and Department of Psychology, University College London, London WC1N 3AR, United Kingdom
}

\begin{abstract}
Skilled interactions with new environments require flexible changes to the transformation from somatosensory signals to motor outputs. Transcortical reflex gains are known to be modulated according to task and environmental dynamics, but the mechanism of this modulation remains unclear. We examined reflex organization in the sensorimotor cortex. Subjects performed point-to-point arm movements into predictable force fields. When a small perturbation was applied just before the arm encountered the force field, reflex responses in the shoulder muscles changed according to the upcoming force field direction, indicating anticipatory reflex gain modulation. However, when a transcranial magnetic stimulation (TMS) was applied before the reflex response to such perturbations so that the silent period caused by TMS overlapped the reflex processing period, this modulation was abolished, while the reflex itself remained. Loss of reflex gain modulation could not be explained by reduced reflex amplitudes nor by peripheral effects of TMS on the muscles themselves. Instead, we suggest that TMS disrupted interneuronal networks in the sensorimotor cortex, which contribute to reflex gain modulation rather than reflex generation. We suggest that these networks normally provide the adaptability of rapid sensorimotor reflex responses by regulating reflex gains according to the current dynamical environment.
\end{abstract}

Key words: reflex gain modulation; anticipation; dynamical interaction; sensorimotor cortex; transcranial magnetic stimulation; arm movement control

\section{Introduction}

To interact successfully with the external environment, the dynamical properties of the musculoskeletal system, such as joint torques and stiffnesses, should be appropriately regulated for the dynamical interactions that will occur. It is, therefore, important to understand how the CNS regulates musculoskeletal dynamics according to expected dynamical interactions. Several studies suggest that musculoskeletal dynamics during voluntary movements are modified by automatic or "reflex" transformations from somatosensory signals to motor outputs (Lacquaniti and Maioli, 1989; Hore et al., 1990; Goodin and Aminoff, 1992; Bennett et al., 1994; Wang et al., 2001; Gomi et al., 2002), as well as by feedforward processes (Burdet et al., 2001; Franklin et al., 2003), according to relevant dynamic properties of the external environment. It remains unclear, however, how the CNS controls and implements this modulation.

Long-latency stretch reflexes in the upper limb muscles are believed to involve the primary motor cortex (M1) (Conrad et al.,

\footnotetext{
Received Sept. 13, 2005; revised July 2, 2006; accepted July 24, 2006.

We gratefully thank J. C. Rothwell for comments and suggestions, N. Saijo for technical support, and T. Moriya and S. Katagiri for encouragement and support.

Correspondence should be addressed to Toshitaka Kimura, NTT Communication Science Laboratories, Nippon Telegraph and Telephone Corporation, 3-1, Morinosato Wakamiya, Atsugi-shi, Kanagawa 243-0198, Japan. E-mail: t-kimura@idea.brl.ntt.co.jp.

DOI:10.1523/JNEUROSCI.3886-05.2006

Copyright $\odot 2006$ Society for Neuroscience $\quad 0270-6474 / 06 / 269272-10 \$ 15.00 / 0$
}

1984; Day et al., 1991). Such transcortical reflexes are modulated according to ongoing tasks (Johnson et al., 1993; Dietz et al., 1994) or instruction (Bonnet, 1983; Sullivan and Hayes, 1987; Yamamoto and Ohtsuki, 1989; Goodin and Aminoff, 1992). In particular, primate studies have demonstrated that the neural responses in M1 to a mechanical stretch of the upper limb muscles are strongly task dependent (Evarts and Tanji, 1974, 1976). Such studies do not show how modulation of reflexive outputs from M1 is achieved. Moreover, relatively few studies have investigated whether the reflex organization is modulated according to the dynamics of the external environment while the subject's motor task remains constant.

Studies of voluntary movement show that interneuronal networks within M1 are modulated in a task-dependent manner (Mathis et al., 1998, 1999; Hess et al., 1999; Tinazzi et al., 2003). For example, the duration of a long-lasting electromyogram (EMG) suppression [silent period (SP)] caused by transcranial magnetic stimulation (TMS) was longer during simple than complex manual tasks (Tinazzi et al., 2003) and during exploratory than visually guided movement (Hess et al., 1999). These findings suggest that task-dependent modulation involves an inhibitory interneuronal activation in M1, because this inhibitory activation is thought to be the major origin of SP (Ziemann et al., 1993; Brasil-Neto et al., 1995; Nakamura et al., 1997). However, the role of these inhibitory circuits in nonvoluntary, adaptive sensorimotor responses such as the transcortical reflex has not been dem- 


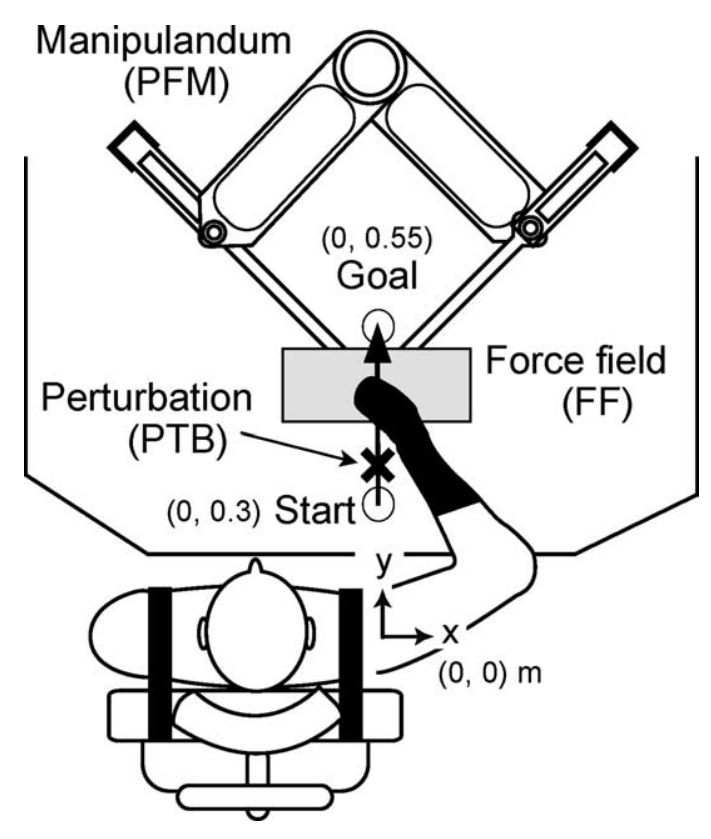

Figure 1. Experimental setup. By using a PFM system, subjects performed point-to-point arm movements forward from the body. During the later part of movements, a leftward (L-FF) or rightward (R-FF) force field was applied in addition to null force (N-FF).

onstrated directly, because previous SP studies focused on voluntary actions.

We therefore hypothesized that interneuronal activity in M1 would be involved in reflex gain modulation according to the dynamical environment. We here applied TMS over M1 before the stretch reflex response induced by mechanical perturbations, so that the SP partially overlapped the reflex response. This operation would disrupt M1 interneuronal activity during the reflex processing period. We therefore investigated the contribution of M1 interneuronal activity to reflex modulation during a simple voluntary reaching task performed in a range of different dynamical environments. A part of this study has been presented previously in abstract form (Kimura et al., 2004).

\section{Materials and Methods}

Subjects. Eight right-handed healthy male subjects (age range, 22-35 years) participated in this study. The subjects had no known history of neurological disorder and gave informed consent. This study was approved by the local ethical committee.

Experimental setup and tasks. The subjects were comfortably seated in a chair set in front of a parallel link drive air-magnet floating manipulandum (PFM) (Gomi and Kawato, 1996) with their trunk held by straps so that the right shoulder joint was positioned in the origin $([x, y]=[0,0])$ of a task coordinate system, in which $x$ - and $y$-axes indicate the rightward and forward direction away from the body, respectively (Fig. 1). The right forearm was covered by a custom-molded thermoplastic cuff closely coupled to the handle of the PFM and supported against gravity by a horizontal beam-attached handle, which immobilized the wrist joint in supination and supported the forearm. Consequently, arm motion was limited to two degrees of freedom at shoulder and elbow joints. The subject's hand and forearm were masked by a screen placed $0.3 \mathrm{~m}$ above the PFM table. The PFM was controlled by a digital servo at $2 \mathrm{kHz}$. The handle position of PFM and the external force exerted at the handle were measured by a position encoder (409,600 pulses/revolution) and a force sensor (resolution, $0.006 \mathrm{~kg}$ ), respectively, at a sampling rate of $500 \mathrm{~Hz}$.

The subjects were instructed to perform point-to-point movements (duration of $1.5 \mathrm{~s})$ from a proximal start $([0,0.3] \mathrm{m})$ to a distal goal $([0$, $0.55] \mathrm{m}$ ) in the horizontal plane with as straight a path as possible (Fig. 1). The movement was executed with the guidance of beeping sounds to indicate the desired duration and a visual guide marker moved by a minimum jerk trajectory. One of three force fields was imposed in the latter part of the movement (0.42-0.53 $\mathrm{m}$ in $y$-direction). These were a null force field (N-FF), and a leftward (L-FF) or rightward (R-FF) trapezoidal-shaped force field (peak of $7 \mathrm{~N}$ ). We applied a transition phase so that force changed gradually over the first and last $0.02 \mathrm{~m}$ of the force field zone. Information for each force field condition was displayed on the screen as a symbol (L-FF/R-FF, arrow; N-FF, rectangle) beginning $2 \mathrm{~s}$ before the go signal for movement. The order of force fields was randomized for each subject. The start and goal positions, visual movement guide, and directional cues were all projected onto a horizontal screen placed directly above the work surface. The current hand position was also displayed on the screen before movement onset, was extinguished at the time of the go signal, and was redisplayed 1.5 s later, at the end of the movement. Therefore, subjects had no visual feedback of hand position during movements. Before the experimental conditions, subjects practiced moving through force fields that were randomized from trial to trial (several times for each force field condition) without any mechanical perturbations, so as to learn to produce straight arm trajectories. To confirm that the subjects had truly learned to control their movements appropriately for each experimental force field direction, three "after-effect" trials, in which a force field was cued but unexpectedly omitted, were performed at the end of the experiment. Subjects were asked not to compensate for these "catch" force fields.

Mechanical perturbations and TMS. A half-sine wave brief force perturbation (PTB) (peak amplitude of $20 \mathrm{~N}$, duration of $50 \mathrm{~ms}$ ) in the left (L-PTB) or right (R-PTB) direction was applied during the movement, but before the arm encountered the force fields. The perturbation occurred $50 \mathrm{~ms}$ after the hand passed through a trigger location, $0.03 \mathrm{~m}$ forward from the start position. The perturbation was designed to be small enough not to disturb ongoing arm movement. The L-PTB and R-PTB primarily stretched the extensor and flexor muscles around the shoulder joint, respectively, and were designed to elicit stretch reflex responses in these muscles.

In one-half of all trials at random, we applied a single TMS over the primary motor cortex (M1) before the perturbation, to examine the effect of TMS on the reflex responses. TMS was delivered to the left M1 using a Magstim 200 stimulator (Magstim Company, Whitland, UK) connected to a focal figure-of-eight coil with external loop diameters of 9 $\mathrm{cm}$ (maximum output of $1.5 \mathrm{~T}$ ) to generate induced currents in the posterior-to-anterior direction. The coil was positioned so as to optimally evoke a motor-evoked potential (MEP) in the pectoralis major muscle. TMS intensity was determined so that TMS caused a clear period (>100 ms) of EMG suppression after the MEP (i.e., SP) in the pectoralis muscle during the movements. To achieve this SP, we adjusted TMS intensity for every subject to a level between 110 and 120\% of resting motor threshold (MT). The MT was defined as the minimal intensity necessary to elicit an MEP of $>50 \mu \mathrm{V}$ (at least three of five successive times) in the resting pectoralis muscle. The SP was checked by monitoring EMG signals on an oscilloscope at the beginning of the experiments. We applied TMS when the hand moved through a trigger position $0.03 \mathrm{~m}$ forward from the start position. TMS thus occurred $50 \mathrm{~ms}$ before the onset of perturbation. Thus, the mechanical perturbation and the resulting reflex response both occurred during the SP after TMS. The TMS coil and subject's head position were maintained throughout the experiment by a custom-made coil holder with adjustable links, a head rest and a chin-forehead support.

The experiment was designed as a factorial combination of three force fields (L-FF, R-FF, N-FF), three perturbations (L-PTB, R-PTB, no perturbation), and two TMS conditions (with TMS, without TMS). The subjects randomly performed 12 trials in each combination, giving a total of 216 trials. Subjects were instructed not to resist any effects of either the perturbation or the TMS on the arm trajectory.

A control task was used to determine each subject's voluntary reaction time. This task was as above, except that subjects performed voluntary, assistive arm movements in the same direction as the perturbation, in each force field condition. Subjects were asked to voluntarily move their hand in the same direction as the perturbation direction as quickly as possible (i.e., rightward hand movement to rightward perturbation or 
Table 1. Measures of background muscle activity and responses to perturbation and to TMS

\begin{tabular}{|c|c|c|c|c|c|c|c|c|}
\hline & & $\mathrm{BGA}(\mu \mathrm{V})$ & $\operatorname{tmsBGA}(\mu \mathrm{V})$ & SR-on (ms) & SR-term (ms) & tmsSR-on (ms) & tmsSR-term (ms) & SP-term (ms) \\
\hline \multirow[t]{2}{*}{ Flexor } & $\mathrm{R}-\mathrm{FF}$ & $10.3 \pm 0.6$ & $14.2 \pm 1.4$ & $49.4 \pm 6.3$ & $126.1 \pm 10.6$ & $76.8 \pm 13.4$ & $162.0 \pm 16.6$ & $124.8 \pm 10.6$ \\
\hline & L-FF & $10.1 \pm 0.9$ & $13.2 \pm 1.6$ & $50.7 \pm 6.3$ & $122.1 \pm 11.8$ & $78.5 \pm 14.7$ & $160.1 \pm 17.2$ & $130.1 \pm 13.5$ \\
\hline \multirow[t]{2}{*}{ Extensor } & $\mathrm{R}-\mathrm{FF}$ & $9.8 \pm 2.5$ & $14.9 \pm 3.0$ & $65.9 \pm 10.2$ & $154.3 \pm 13.3$ & $78.4 \pm 14.2$ & $162.8 \pm 14.1$ & $133.8 \pm 17.3$ \\
\hline & L-FF & $11.1 \pm 3.1$ & $15.8 \pm 2.7$ & $63.5 \pm 12.2$ & $151.8 \pm 15.1$ & $79.7 \pm 13.7$ & $161.3 \pm 12.4$ & $129.3 \pm 19.6$ \\
\hline
\end{tabular}

The mean ( \pm SE) background muscle activity in the control trials (BGA) and the mean ( \pm SE) muscle activity in the trials with TMS-only (tmsBGA), the mean ( \pm SD) onset (SR-on) and termination (SR-term) time of induced stretch reflex response after the perturbation, the mean ( \pm SD) onset (tmsSR-on) and termination (tmsSR-term) time of the response with TMS, and the mean ( \pm SD) termination time of silent period (SP-term) after TMS. The BGA and tmsBGA were calculated over the same window as the reflex response in each condition (with or without TMS). Note no significant difference between the force fields in each parameter.

leftward movement to leftward perturbation). Subjects performed six trials in each combination of force field and perturbation. The order of force fields and perturbation directions was randomized. The reaction time was determined by detecting the onset of a burst of voluntary EMG in the ensemble-averaged EMG waveform for each combination of force field and perturbation.

EMG measurement. Surface EMG activity was recorded from the bellies of the two muscles around the shoulder joint: shoulder flexor (pectoralis major) and extensor (deltoid posterior), using pairs of $\mathrm{Ag}-\mathrm{AgCl}$ electrodes (diameter, $10 \mathrm{~mm}$ ) with a center-to-center interelectrode distance of $20 \mathrm{~mm}$. Analog EMG signals were amplified with a bioelectric amplifier (MME-3132; Nihon Kohden, Tokyo, Japan) and digitized at a sampling rate of $2 \mathrm{kHz}$. The digitized EMG signals were full-wave rectified, and then low-pass filtered at $100 \mathrm{~Hz}$ to reduce the jaggy component. We first detected an onset and termination time of reflex response by visual inspection from the ensemble-averaged waveform in each combination of force field and muscle (Table 1). We separately confirmed interrater reliability by asking a second rater to estimate latencies on a subset of trials from eight subjects and from two force fields. This showed strong correlation coefficients between two raters on detections of each onset and termination time in each muscle (all $r>0.78, p<0.01$; difference in detection time, mean $\pm \mathrm{SD}, 4.9 \pm 3.5 \mathrm{~ms})$. We then measured the mean EMG value for each signal in each trial during the period between reflex onset and termination period. Background muscle activity (BGA) was calculated as the EMG activity over the same time period in trials in which neither perturbations nor TMS occurred. The EMG activity after the perturbation minus the BGA was taken as the reflex response. A similar procedure was used to give an independent estimate of the reflex response component when applying TMS: here, we subtracted EMG activity with TMS-only (tmsBGA) from that with the perturbation and TMS. The termination time of SP (i.e., the time that the EMG activity returns to the BGA level) was also determined by visual inspection from the ensemble-averaged waveform in TMS-only trials.

Dynamic and kinematic data processing. The procedure for estimating dynamic and kinematic parameters has been described in detail previously (Gomi and Kawato, 1997; Gomi and Osu, 1998). Briefly, muscle generated arm-joint torque, $\tau_{\text {in }}$, can be estimated by using the following dynamical equation:

$$
I(q) \ddot{q}+H(\dot{q}, q)=\tau_{\text {in }}+\tau_{\text {ext }},
$$

where $q, \dot{q}$, and $\ddot{q}$ denote angular position vector of shoulder and elbow joints, its velocity and acceleration vectors, respectively. Joint angle was obtained from the handle position using the kinematic relationship. Joint velocity and acceleration were calculated by first- and second-order time differentiations of each joint angle, respectively. $\tau_{\text {ext }}$ denotes the torque vector transmitted from the PFM (i.e., external torque), calculated by $\tau_{\text {ext }}=J^{T} F_{\text {ext }}$, where $J$ denotes Jacobian matrix of two-link human arm kinematics, and $F_{\text {ext }}$ denotes the external force measured at the handle. $I$ and $H$ denote the inertial matrix and Coriolis centrifugal force vector, respectively, whose internal parameters were estimated in advance for each subject by using a procedure reported previously (Gomi and Osu, 1998).

Statistical analysis. One-way ANOVA and Bonferroni-corrected post hoc comparisons were used to test each subject's data individually for differences in the BGA and tmsBGA among the force field conditions (L-FF, R-FF, and N-FF). A significant difference between conditions was found for at least one muscle in most ( six of eight) subjects using one-way ANOVA with trials as a random factor. Bonferroni comparisons showed that BGA and tmsBGA in the N-FF were significantly smaller than in $\mathrm{L}-\mathrm{FF}$ and R-FF for most of these ANOVAs. In contrast, there were no significant differences in the BGA and tmsBGA between the L-FF and $\mathrm{R}-\mathrm{FF}$ in both muscles. Because the amplitude of reflex responses generally depends on the background muscle activity level (Matthews, 1986), it is inappropriate to directly compare the reflex response across conditions that have different BGA. We therefore compared reflexes in the L-FF to those in the R-FF, rather than comparing either of the imposed force fields to the null (N-FF) condition. That is, our analysis focuses on how reflexes are modulated according to force field direction, rather than force field presence. Accordingly, we used two-way repeated-measures ANOVA with factors of force field direction (L-FF/R-FF) and muscle (flexor/extensor), to test for significant differences in reflex responses between force field directions and between agonist and antagonist muscles. The significance level was set at $p<0.05$. Bonferroni-corrected post hoc comparisons were used to follow up significant effects. For the principal analyses reported, the dependent variables were the means of each subject's trials in each condition. Subjects were treated as a random factor. For some subsidiary analyses in the control experiments in which the number of subjects was low, we instead analyzed individual trials, and pooled the data across subjects. These few cases are described in the text as pooled analyses.

\section{Results \\ Changes in reaching behavior attributable to expected force fields}

The thick lines in Figure $2 \mathrm{~A}$ demonstrate an example of the mean hand trajectories in R-FF (black) and L-FF (gray) trials. When the force fields were imposed, the hand trajectories were distorted in the direction of the imposed field, compared with the N-FF trajectory (dotted line). Trajectories in after-effect catch trials (thin lines) done at the end of experiment, in which the force field was cued but not applied, clearly deviated in the direction opposite to the cued field. The bar graph on the right of Figure $2 \mathrm{~A}$ shows hand curvature in each condition. Hand curvature was defined as the positional deviation at the end boundary of the cued force field ( $y=0.53 \mathrm{~m}$ ) in the $x$ direction from a straight line connecting between the start and goal positions. The values of hand curvatures in after-effect trials $(\mathrm{aR}, \mathrm{aL})$ were clearly opposite in sign to those in normal trials ( $\mathrm{R}, \mathrm{L})$, and there were significant differences between after-effect and normal trials (both force field directions, $p<0.001$, paired $t$ tests). This suggests that the subjects learned to move through the applied fields by anticipatorily selecting an internal model that would generate appropriate motor commands. In addition, the existence of a clear after-effect suggests that the distortions of hand trajectories in the R/L-FF direction resulted from insufficient feedforward compensation for the imposed field, rather than reactive correction.

Figure $2 B$ demonstrates an example of the hand position profiles in the $y$-axis (anteroposterior) direction, the hand force profiles in the $x$-axis (left/right) direction, and the EMG waveforms of the shoulder flexor and extensor muscles during arm movements without TMS/PTB. Because the flexor muscle exerts leftward hand force in this arm configuration, its activity increased to exert an increased leftward hand force so as to maintain a 

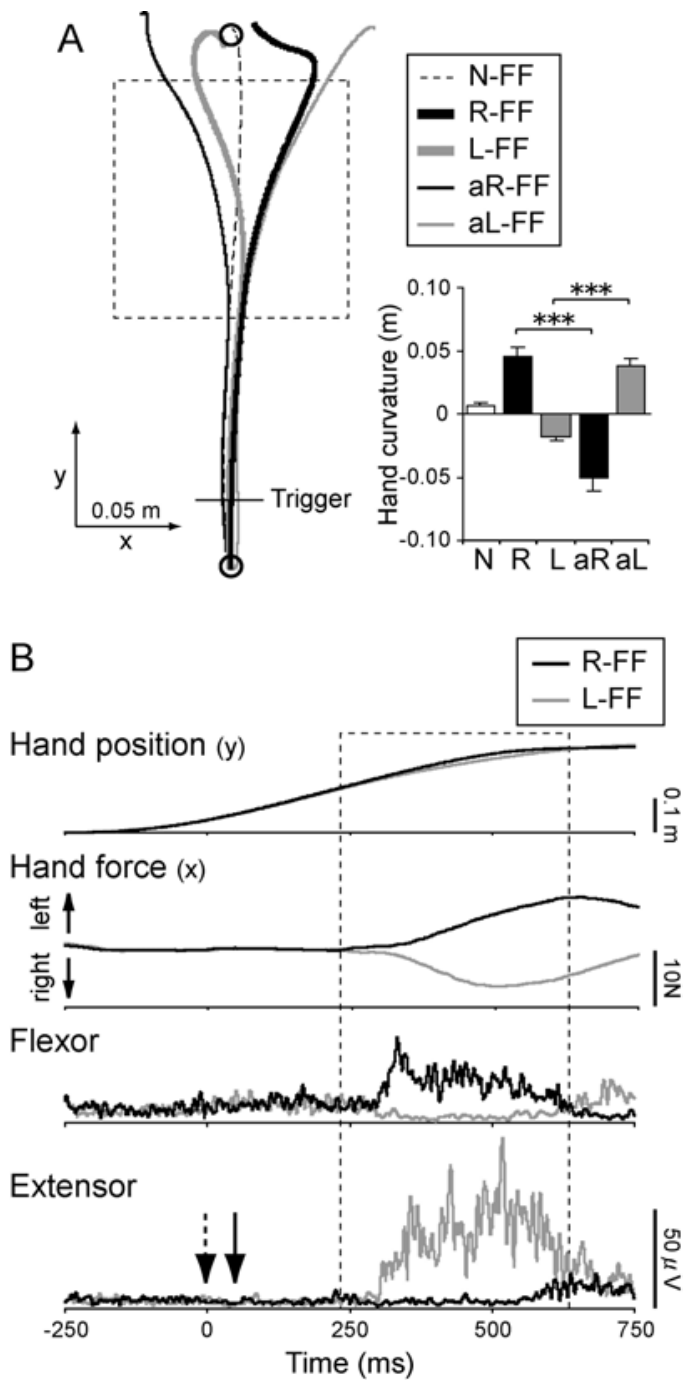

Figure 2. Movement and EMG patterns with different force fields. A, Typical example of hand trajectories (left panel) and each hand curvature (right panel) in the right/left direction in the normal ( $N-F F, R-F F$, and L-FF) and after-effect (aR-FF and aL-FF) trials with different force fields. The rectangular dashed area is force field location. The traces in the normal trials are the average of 12 trials. The traces in the after-effect trials are the average of 3 trials. In after-effect trials, either direction of force field was unexpectedly removed. Each bar in the right graph represents the mean and SE of hand curvature in the right/left direction for each condition. The asterisks express a significant difference between the normal and after-effect trials for each field (*** $<0.001)$. B, An example of the hand position profiles in the $y$-axis direction, the hand force profiles in the $x$-axis direction (upward is left direction), and the EMG waveforms in the shoulder flexor and extensor muscles during arm movements in the R-FF (black line) and L-FF (gray line) conditions. The data show results from a typical subject in baseline conditions with neither perturbation nor TMS. Each trace is the average of 12 trials. The rectangular dashed area denotes the force field period. Time 0 corresponds to a position trigger $(0.03 \mathrm{~m}$ from the start position) shown in Figure $2 A$. The downward arrows in the bottom graph represent the time at which transcranial magnetic stimulation (dashed arrow) and perturbation (solid arrow) would be applied in other conditions as appropriate. Note that the muscle activity before the arm encountered the force fields was almost same between the R-FF and L-FF conditions.

straight hand trajectory when the hand encountered the R-FF. In contrast, the extensor muscle activity increased in the L-FF condition to exert rightward hand force.

However, the EMG activities before encountering the force fields did not vary predictively and were almost identical for the two force field conditions. Indeed, paired $t$ test showed no significant difference in the mean BGA values in both muscles (both muscles, $p>0.09$ ) (Table 1). We used similar analyses to inves- tigate whether kinematic responses to the perturbations differed between the two force field directions, at the same point in the trajectory. Figure $3 \mathrm{~A}$ demonstrates typical waveforms for hand position in the $x$-axis (left/right) direction, shoulder joint angular velocities in the flexion/extension direction, and EMG in the shoulder flexor and extensor muscles, when the R-PTB (upper panels) or L-PTB (lower panels) was applied (thick lines). There was no significant difference in two kinematic parameters between the two field conditions in the initial part (0-100 ms after the perturbation) of the movement (all $p>0.18$, paired $t$ tests). The specific values compared were as follows: $x$ hand displacement (R-FF for R-PTB, mean \pm SE, $0.016 \pm 0.0007 \mathrm{~m}$; L-FF, $0.017 \pm 0.0017 \mathrm{~m}$; R-FF for L-PTB, $-0.015 \pm 0.0008 \mathrm{~m}$; L-FF, $-0.014 \pm 0.0012 \mathrm{~m}$ ), shoulder joint angular velocities (R-FF for $\mathrm{R}-\mathrm{PTB},-0.23 \pm 0.013 \mathrm{rad} / \mathrm{s} ; \mathrm{L}-\mathrm{FF},-0.24 \pm 0.015 \mathrm{rad} / \mathrm{s}$; R-FF for $\mathrm{L}-\mathrm{PTB}, 0.27 \pm 0.020 \mathrm{rad} / \mathrm{s} ; \mathrm{L}-\mathrm{FF}, 0.26 \pm 0.033 \mathrm{rad} / \mathrm{s}$ ). Therefore, changes in reflex amplitude could not be attributed to differences in background muscle activity or physical parameters of the limb.

\section{Changes in reflex responses according to expected force field directions}

The R-PTB and L-PTB induced stretch reflex responses in both shoulder flexor and shoulder extensor muscles, respectively. These reflex responses were visible 50-150 ms after the perturbation. The mean onset (SR-on, both muscles, $p>0.09$ ) and termination (SR-term, $p>0.16$ ) time of reflex responses did not differ significantly between the two force field directions (Table 1). However, the reflex response amplitude varied according to the direction of the expected force field (Fig. $3 A$, thick black and gray lines). The mean reflex amplitudes in both muscles are shown in Figure 3B. Two-way repeated-measures ANOVA with factors of force field direction (L-FF/R-FF) and muscle (flexor/ extensor) showed no significant main effect of force field direction or muscle. The interaction between these factors was highly significant, however $(p<0.001)$. Subsequent, Bonferronicorrected post hoc comparisons demonstrated that the flexor reflex response was larger in the R-FF condition $(p<0.01)$ than in the L-FF condition, whereas the extensor reflex was, in contrast, larger in the L-FF condition than in the R-FF condition $(p<$ $0.01)$. These symmetric modulations of reflex amplitude clearly depended on whether the muscle would successfully counteract the direction of the force field that would soon be encountered (Fig. 2 B). These results indicate that stretch reflex gain is anticipatorily modulated depending on expected force field direction.

The duration of these reflexes appeared somewhat longer than that reported by others (Day et al., 1991; Lewis et al., 2004). This may be because the subject's shoulder and elbow were actively moving at the time that the force perturbation was applied to the hand. Therefore, we confirmed that these EMG responses were genuine reflexes rather than voluntary reactions as follows. All subjects performed a separate control task in which they were specifically asked to make assistive voluntary reactions to identical perturbations as fast as possible (i.e., leftward movement in response to L-PTB, rightward movement in response to R-PTB). This control task was designed to give the fastest possible latency for voluntary reactions, so as to separate voluntary reaction from reflex response on the basis of latency. The thin lines in Figure $3 \mathrm{~A}$ show assistive voluntary responses in this control task. Note here that no reflex response was induced in the agonist muscle in this voluntary reaction task, because the agonist muscle was not stretched by the perturbation. In contrast, a reflex response was now observed in the antagonist muscle. Comparison of reflex and voluntary reactions showed that reflex (Fig. $3 A$, thick lines) and 
A
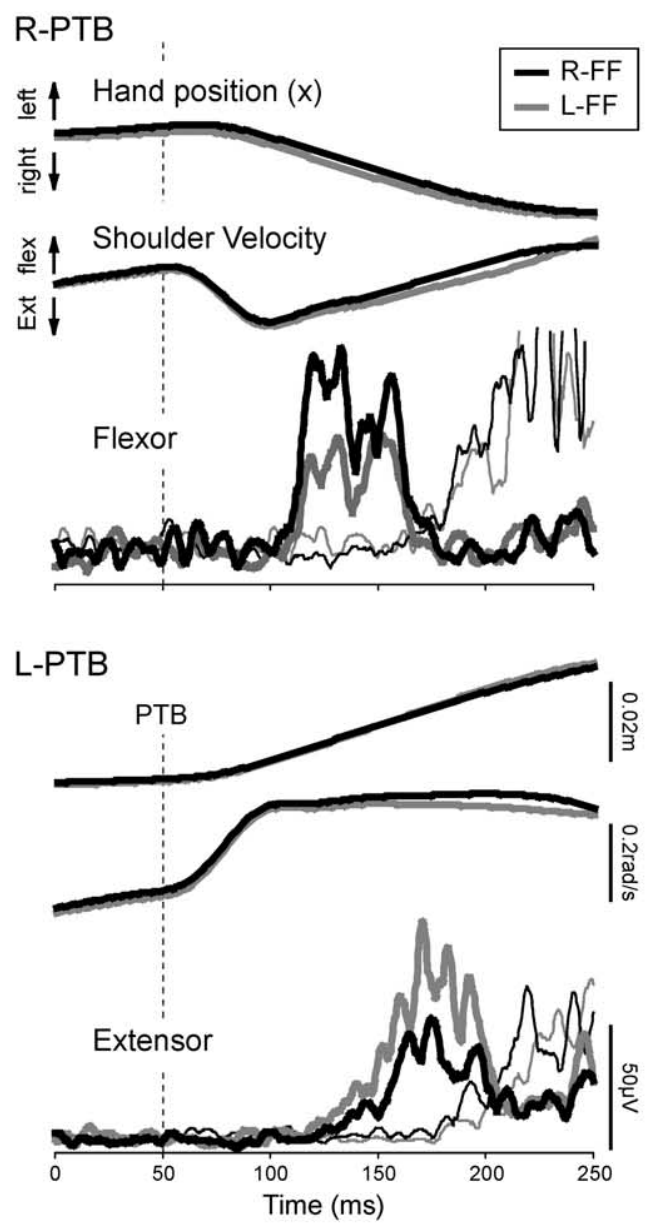

B
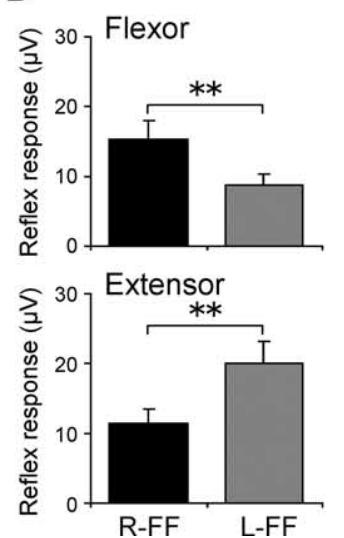

C
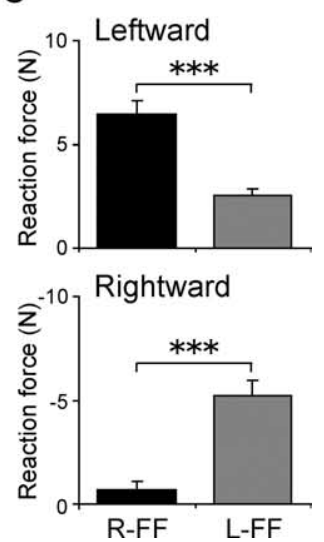

Figure 3. Differences in stretch reflex responses and hand reaction forces between the force fields. $\boldsymbol{A}$, An example of the waveforms of hand position in the $x$-axis (right/left) direction, shoulder joint angular velocity in the flexion/extension direction, and EMG in the shoulder flexor and extensor muscles when a mechanical perturbation was applied (thick lines). The rightward (R-PTB; top panels) and leftward (L-PTB; bottom panels) perturbations applied at 50 ms indicated by the vertical dashed line induce reflex responses in the flexor and extensor muscles, respectively. The thin lines indicate the EMG waveforms in a control task in which the subject was instructed to make voluntary assistive reactions to perturbations. For example, the voluntary response to a leftward perturbation is used as a control for the reflex response to a rightward perturbation. Note the clear temporal separation between reflex and voluntary responses. $\boldsymbol{B}$, Comparisons of reflex responses in the flexor and extensor muscles between the L-FF and $\mathrm{R}-\mathrm{FF}$ conditions. Each bar represents the mean and SE for each muscle. The asterisks express a significant difference between the force fields $\left({ }^{* *} p<0.01\right.$ ). Note that the reflex amplitudes in the flexor and extensor muscles were larger in the R-FF and in the L-FF condition, voluntary assistive (thin lines) EMG waveforms were markedly different in latency and shape. There was little overlap between reflex EMG and voluntary EMG activity (flexor reflex termination time, mean $\pm \mathrm{SD}, 124.1 \pm 11.2 \mathrm{~ms}$; the extensor, $153.1 \pm$ $14.2 \mathrm{~ms}$; flexor voluntary reaction time, $114 \pm 11.3 \mathrm{~ms}$; the extensor, $122 \pm 11.9 \mathrm{~ms}$ ). Therefore, the voluntary contribution for correction to the perturbation was judged to be negligible.

\section{Functional contribution of reflex responses to arm motor control}

To assess the contribution of reflex gain modulation to the functional output of the arm system, we evaluated rightward and leftward reaction forces produced at the hand by the L-PTB and R-PTB, respectively, after the reflex response (200-250 ms after the perturbation). Figure $3 C$ shows the mean peak values of hand reaction forces. The leftward reaction force produced by the R-PTB (top panel) was significantly larger in the R-FF than in the L-FF condition $(p<0.001)$. The rightward reaction force produced by the L-PTB (bottom panel) was, in contrast, larger in the L-FF condition $(p<0.001)$. These force field-dependent changes in reaction forces reflect differences in reflex responses in the shoulder muscles between the force fields, because there was no difference in the BGA levels between the force fields as mentioned (see above). That is, the strong flexor reflex in the R-FF condition contributed to a larger leftward reaction force in that condition, whereas the larger extensor reflex in the L-FF condition contributed to a larger rightward force in that condition. This indicates that the reflexes contributed functional forces to resist the imposed force fields.

We suggest that such behavior of reaction forces may reflect an active control of limb stiffness (resistance to the positional shift) by the CNS, in a manner appropriate for the current environment. We cannot calculate the limb stiffness from the current results, because we measured the responses to the perturbations in only two $(+x,-x)$ directions. Conventional measures of limb stiffness at the hand require sampling responses to many directional perturbations in the horizontal plane (Mussa-Ivaldi et al., 1985; Gomi et al., 1998). However, because the perturbations caused similar hand displacements between the force fields (see above), the hand reaction force is approximately proportional to instantaneous hand stiffness in that direction. We therefore suggest that leftward hand stiffness was selectively increased in the L-FF condition and the rightward stiffness was selectively increased in the R-FF condition. From the functional viewpoint, this behavior of hand stiffness is quite reasonable, because it acts to prevent distortion of hand trajectories toward force field direction. Thus, reflex gain modulation may contribute to regulate hand stiffness according to the expected force field direction.

\section{Disappearance of reflex gain modulation by previous TMS}

To examine the involvement of interneuronal activity within M1 in reflex gain modulation, we applied TMS over the left M1 $50 \mathrm{~ms}$ before the perturbation. The TMS intensities used were $77.1 \pm$ $3.5 \%$ (mean $\pm \mathrm{SD}$ ) of the maximum stimulator output capacity, corresponding to $110-120 \%$ of each subject's MT in the resting pectoralis. As shown in Figure 4A (thin lines, no perturbation

$\leftarrow$

respectively. C, Differences in the hand reaction force after reflex response between the force fields. The leftward and rightward reaction forces were calculated from the R-PTB and L-PTB trials, respectively. Other notations are the same as in $\boldsymbol{B}$. Note that the leftward reaction force was larger in the R-FF condition, and, in contrast, the rightward force was larger in the L-FF condition $(* * * 00.001)$. 
A
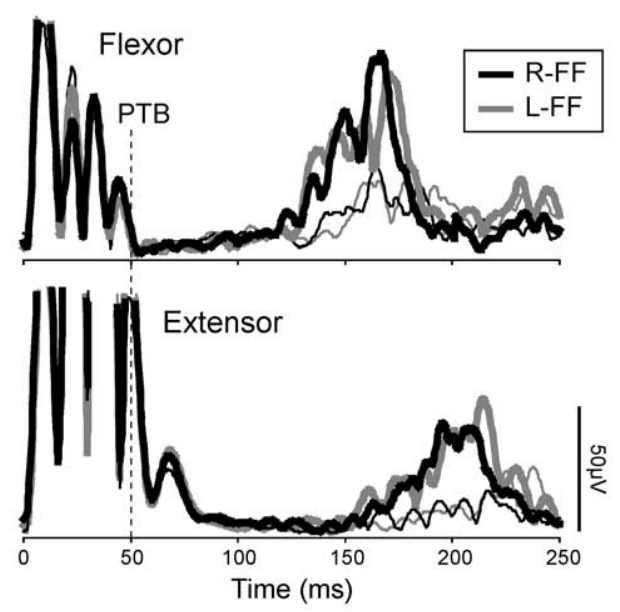

B
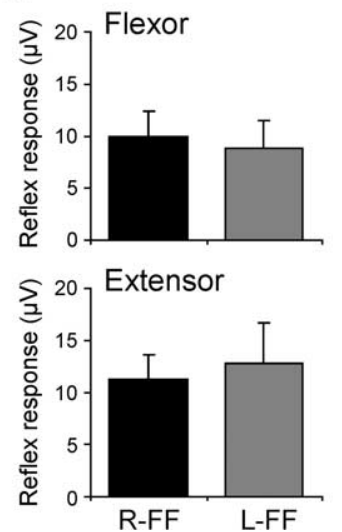

C
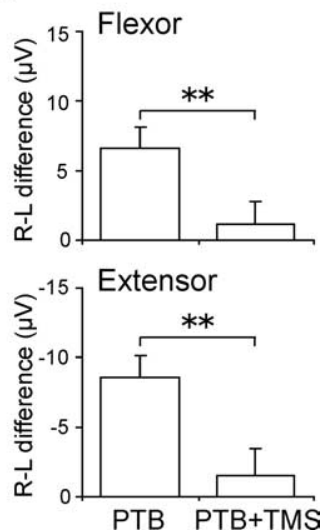

Figure 4. Effect of previous TMS on stretch reflex modulation. $\boldsymbol{A}$, An example of the reflex responses in both muscles (thick lines) when TMS was applied 50 ms before the perturbation (vertical dashed line). The thin lines indicate the waveforms when only TMS was applied. $\boldsymbol{B}$, Comparisons of reflex responses with TMS in both muscles between the force fields. Other notations are the same as in Figure $3 B$. C, Comparisons of the difference in reflex response between the fields ( $R-F F$ minus L-FF). The asterisks express a significant difference between the force fields $\left({ }^{* *} p<0.01\right.$ ). Note that the differences in reflex responses between the force fields were eliminated by applying previous TMS, whereas the reflex responses themselves were still clearly present.

conditions for R-FF and L-FF), TMS elicited first a transient MEP, followed by prolonged EMG suppression (i.e., SP) and a subsequent EMG increase (i.e., post-SP rebound activity) (Triggs et al., 1993). Note here that an artifact of the TMS pulse was overlapped to the initial part of the MEP because of the short latency of conduction from the cortex to the shoulder muscle ( $\sim 10 \mathrm{~ms}$ ) (Bawa et al., 2004) coupled with the effect of the lowpass filter (see above). The mean termination time of the SP (SP-term) without the perturbation exceeded $100 \mathrm{~ms}$ in both muscles (Fig. $4 A$, thin lines). There was, however, no significant difference in either the SP-termination time (both muscles, $p>$ 0.23 ) or the background activities of no perturbation trials with $\mathrm{SP}$ and rebound activity (tmsBGA) (both muscles, $p>0.10$ ) between the force fields (Table 1).

We therefore suggest that the reflex response to mechanical perturbations substantially overlapped the disruption to the cortical interneuronal network marked by the SP. First, the latency of the reflex response in normal (without TMS) condition was $\sim 50$ $\mathrm{ms}$ after the perturbation, and $100 \mathrm{~ms}$ after TMS. This falls within the SP window (Fig. 3A, Table 1). Second, the latter part of SP is thought to be attributable to activation of inhibitory interneuronal networks within M1 (Ziemann et al., 1993; Brasil-Neto et al., 1995; Nakamura et al., 1997; Chen, 2004). These networks may play a key role in shaping transcortical reflexes under normal conditions.

When both TMS and perturbations were delivered, TMS did not abolish stretch reflex responses to the perturbation (Fig. 4A, thick lines), although it did significantly delay its onset (tmsSRon) and termination (tmsSR-term) times relative to trials without TMS (onset time for the flexor, $p<0.001$; the extensor, $p<$ 0.01 ; termination time for the flexor, $p<0.001$; the extensor, $p<$ $0.05)$. However, the response latency with TMS was clearly earlier than voluntary onset time by $\sim 30 \mathrm{~ms}$ (reflex latency with TMS, mean $\pm \mathrm{SD}, 78 \pm 14 \mathrm{~ms}$; voluntary onset, $118 \pm 12 \mathrm{~ms}$ ). This suggests that the response with TMS include little or no voluntary correction to the perturbation. The response delays after TMS are probably attributable to SP-related inhibitory effects, and recall the delays in voluntary motor output induced by TMS in voluntary reaction tasks (Day et al., 1989).

Most importantly, the differences in reflex responses between the force fields tended to disappear in trials with TMS (thick lines), in contrast to the clear differences seen in trials without TMS (Fig. $3 A, B$ ). Indeed, two-way repeated-measures ANOVA found no significant main effect of force field direction $(p=$ 0.67 ) or muscle ( $p=0.67)$, and no significant interaction between these factors $(p=0.79$ ) (Fig. $4 B$ ). Figure $4 C$ shows the difference between the responses in the two force field conditions (R-FF - L-FF), for TMS and for no-TMS trials. These difference scores were significantly lower in TMS trials than in no-TMS trials (both muscles, $p<0.01$ ). Accordingly, two-way repeatedmeasures (L-FF/R-FF and with/without TMS) ANOVA showed a significant interaction for flexor $(p<0.01)$ and extensor reflexes $(p<0.05)$. These results indicate that TMS-induced disruption to interneuronal activity in M1 during the SP may cause reductions in reflex gain modulation.

To secure this conclusion, however, we should consider whether our results could be an artifactual consequence of two other unavoidable effects of TMS. These are the following: (1) the general reduction in reflex responses in TMS condition, and (2) the effect of muscle twitches generated by TMS and corresponding peripheral changes. To examine these possibilities, we performed two additional control experiments described below.

\section{Control experiment 1: TMS-induced reduction in reflex gain modulation is not attributable to reduced reflex response amplitude}

TMS reduced overall reflex amplitude, as well as reducing fielddependent modulations. To investigate whether the former effect could be responsible for the latter, we investigated reflex gain modulation in the absence of TMS, after small mechanical perturbations. These perturbations evoked reflex responses at least as small as those seen after TMS in the main experiment. Three subjects participated, and a pooled analysis was therefore used. Perturbations were 12.5 or $15 \mathrm{~N}$, in contrast to the $20 \mathrm{~N}$ of the main experiment. To verify that these small perturbations elicited reflexes with amplitudes comparable with those found in the combined TMS and perturbation conditions of the main experiment, the BGA-subtracted reflex response in the control experiment was compared with the tmsBGA-subtracted response of the same subjects in the main experiment. Figure 5 shows the reflex responses in the small PTB (right side) and the normal PTB with TMS (left side) conditions relative to the mean response to the normal PTB in the absence of TMS. Each open and filled 


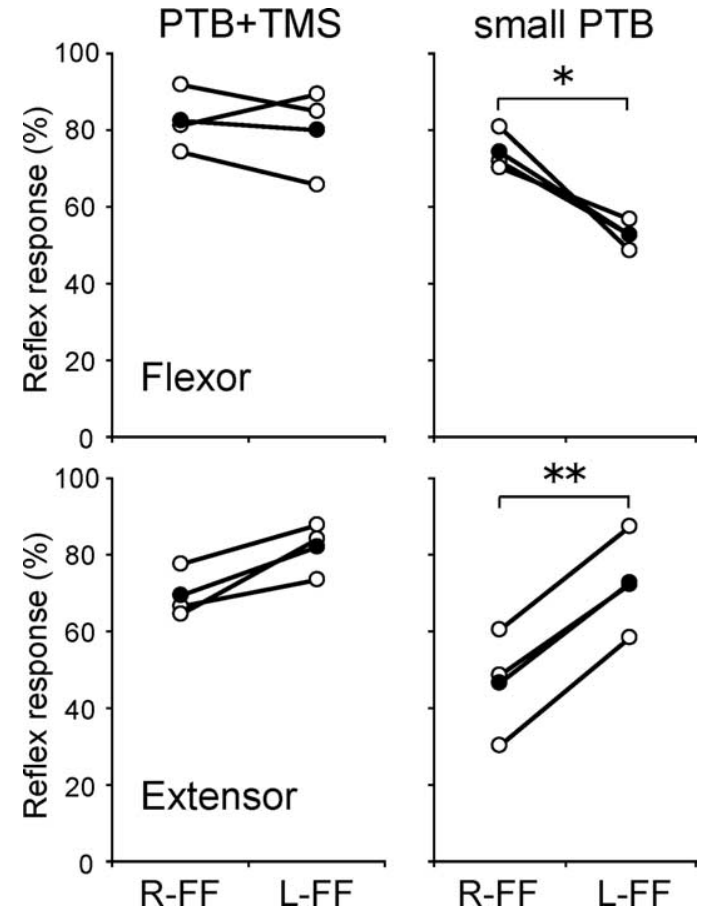

Figure 5. Effect of small perturbation on reflex modulation. The reflex responses in both muscles when normal PTB (20 N) with TMS (left panels) or small PTB (12.5 or $15 \mathrm{~N}$; right panels) was applied. Each open and filled circle indicates the individual and mean values, respectively. Values are represented as percentages of the response induced by the PTB amplitudes used in the main experiment, in no-TMS conditions. The asterisks express a significant difference between the force fields $\left({ }^{*} p 0.05 ;{ }^{* *} p<0.01\right)$. Note that there were significant differences in reflex responses between the force fields even for the smaller reflexes after smaller perturbations, in contrast to the reflexes induced after TMS.

circle represents individual and mean values, respectively. We found that even small perturbations eliciting low reflex amplitudes still showed force field-dependent reflex gain modulation in the absence of TMS. Pooled two-way ANOVA with factors of force field direction (L-FF/R-FF) and muscle (flexor/extensor) showed no significant main effect for either factor, but a highly significant interaction between these factors $(p<0.001)$. Bonferroni-corrected post hoc comparisons demonstrated that the response induced by small PTB in the flexor differed between L-FF and R-FF $(p<0.05)$, as did that in the extensor $(p<0.01)$ (Fig. 3). In contrast, TMS before stronger perturbations in the main experiment reduced both reflex amplitudes and fielddependent reflex gain modulation. These results indicate that the reduction in reflex gain modulation induced by TMS is not merely a by-product of TMS-induced decreases in overall reflex amplitude.

Control experiment 2: Muscle twitch elicited by electrical nerve stimulation does not eliminate reflex gain modulation TMS not only excites the CNS but also causes a sudden twitch of muscles. Twitches of extrafusal fibers could also affect intrafusal fibers in the muscle spindles. Another possible interpretation for the disappearance of reflex gain modulation, therefore, would involve changes in muscle spindle sensitivity (Prochazka, 1989) attributable to the peripheral consequences of TMS. This peripheral sensitivity change would then affect the afferent signals that provide the input to the transcortical reflex, but might not affect cortical processing per se. For example, a TMS-induced muscle twitch might counteract preset sensitivity of muscle spindles and thereby eliminate reflex gain modulation. In other words, reflex
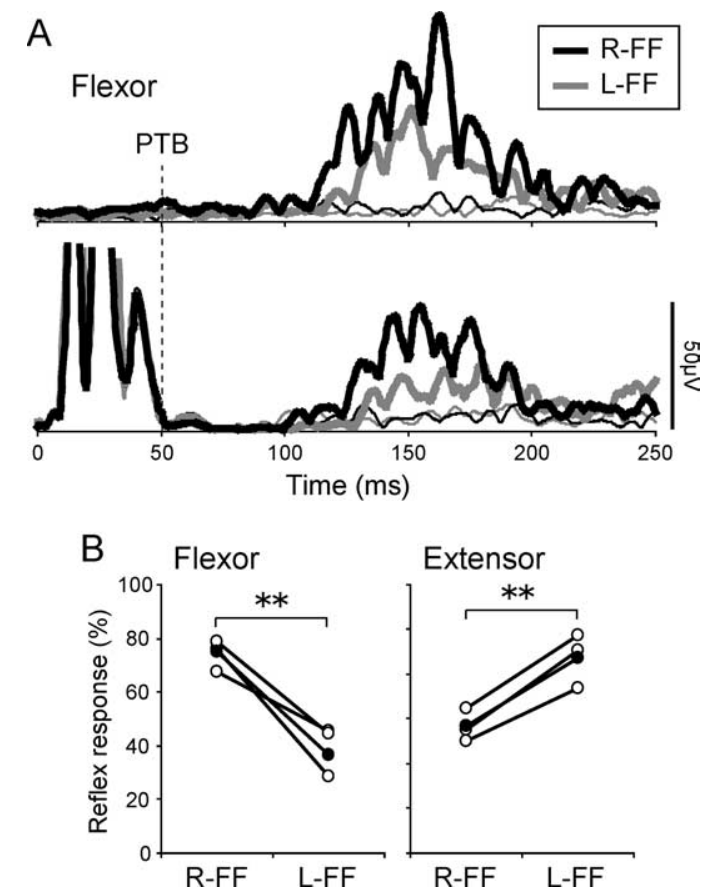

Figure 6. Effect of previous peripheral nerve stimulation on reflex modulation. $\boldsymbol{A}$, An example of the reflex responses (thick lines) in the flexor muscle when a PES at the right brachial plexus was applied (bottom panel) and was not (top panel). The thin lines demonstrate the waveforms without PTB. $\boldsymbol{B}$, The reflex responses in both muscles in PES condition. Other notations are the same as in Figure 5. The asterisks express a significant difference between the force fields ( $\left.{ }^{* *} p<0.01\right)$. Note that even when PES was applied, the modulation of reflex responses according to the cued force fields remained clear.

gain modulation might be occurring peripherally rather than centrally, and might be affected by the muscular rather than cortical, interneuronal effects of TMS.

To control for this possibility, we compared the effects of TMS with effects of an alternative, peripheral stimulation of the motor system, designed to produce similar muscular twitches without involving cortical interneuronal circuits. If these two classes of stimulation suppressed reflex gain modulation equally, the gain suppression effect might be peripheral in origin. Conversely, if TMS stimulation suppressed reflex gain modulation whereas peripheral stimulation did not, the gain suppression effect should be central.

Therefore, percutaneous electrical nerve stimulation (PES) to the right brachial plexus was delivered as a single $1 \mathrm{~ms}$ pulse using a pair of surface electrodes $(\mathrm{Ag} / \mathrm{AgCl}$, diameter of $10 \mathrm{~mm})$ placed adjacent to the clavicle. The stimulus intensity was adjusted so that the evoked force on the manipulandum and the evoked shoulder joint angular acceleration were similar to that induced by TMS in the main experiment. Thus, handle force (TMS, mean $\pm \mathrm{SE}, x=1.70 \pm 0.78, y=-1.74 \pm 0.88 \mathrm{~N}$; PES, $x=$ $2.12 \pm 0.22, y=-2.08 \pm 0.62 \mathrm{~N})$ and shoulder joint angular acceleration (TMS, $3.19 \pm 1.63 \mathrm{rad} / \mathrm{s}^{2}$; PES, $3.40 \pm 1.08 \mathrm{rad} / \mathrm{s}^{2}$ ) were almost comparable between TMS and PES. Three subjects participated in a separate session from the main experiment. Figure $6 \mathrm{~A}$ demonstrates a typical example of reflex responses (thick lines) in the flexor muscle with (lower panel) and without (upper panel) PES. PES produced a period of EMG silence (lower panel), similar to that after TMS. The termination time was $81.8 \pm 14.5$ $\mathrm{ms}$ (mean $\pm \mathrm{SD}$ ) for the flexor and $76.4 \pm 12.8 \mathrm{~ms}$ for the extensor, and did not differ significantly between the force fields (both muscles, $p>0.21$ ). PES did not influence the latency of reflex 
responses (mean $\pm \mathrm{SD}, 53.8 \pm 11.2 \mathrm{~ms}$ for the flexor and $55.1 \pm$ $11.5 \mathrm{~ms}$ for the extensor with PES; mean $\pm \mathrm{SD}, 47.8 \pm 7.5 \mathrm{~ms}$ for the flexor and $52.4 \pm 6.8 \mathrm{~ms}$ for the extensor without PES). PES did affect reflex amplitude, reducing this by 34.1 and $28.0 \%$ for the flexor and extensor, respectively, compared with no-PES condition. Crucially, however, the force field-dependent modulation of reflex gains remained after PES, unlike the TMS condition. Figure $6 B$ demonstrates the reflex responses for the individual (open) and mean (filled) values. These are normalized to the mean response in the PTB only condition, to highlight the differential effects of TMS and PES. Pooled two-way ANOVA with factors of force field direction (L-FF/R-FF) and muscle (flexor/ extensor) showed no significant main effect of force field direction or muscle. Crucially, the interaction between these factors was significant $(p<0.001)$. Bonferroni-corrected post hoc comparisons demonstrated that the reflex response differed between L-FF and R-FF in both flexor $(p<0.01)$, and extensor $(p<$ 0.01 ), despite the effects of PES. Thus, anticipatory reflex modulation remained in the presence of peripheral PES stimulation (Fig. 6B), despite being reduced or abolished by cortical stimulation (Fig. 4). These results mean that the disappearance of reflex gain modulation in the main experiment was not attributable to a purely peripheral effect of TMS.

\section{Discussion}

The present study assessed stretch reflex responses during multijoint arm movements through imposed force fields. Reflex responses were modulated depending on the upcoming force field direction (Fig. $3 B$ ). When we applied TMS over M1 so that the reflex processing overlapped the TMS-induced silent period, we found that this modulation of the reflex response was suppressed (Fig. 4). Control experiments showed that this reduction in modulation was not attributable merely to changes in mean reflex amplitude (Fig. 5) nor to peripheral effects of TMS (Fig. 6). Our study demonstrates both that reflex gains are updated dynamically during the course of aimed arm movements, and that the sensorimotor cortex is involved in this gain-setting. We suggest that anticipatory modulation of transcortical reflex gains may play an important role in coordinating interaction with the external environment.

\section{Flexibility of transcortical reflexes}

The involvement of sensorimotor cortex in long-latency reflex responses in the upper limb muscles has been studied for decades and is now widely accepted (Matthews, 1991). We believe the responses studied here involve transcortical circuits, for three reasons. First, the latency of the reflexes (50 ms and over) was comparable with previous estimates of the "long-loop" reflex response latency in proximal muscles (Marsden et al., 1976b; Colebatch et al., 1979; Yamamoto and Ohtsuki, 1989). Second, the reflexes in our study showed the flexibility and context dependence characteristically associated with cortical responses. Third, the susceptibility of our reflex responses to a cortical intervention (TMS) is consistent with, although not conclusive of, a cortical route.

Several studies have suggested that long-latency stretch reflex gains in the upper limb muscles are modified according to task context (Hallett et al., 1981; Bonnet, 1983; Sullivan and Hayes, 1987; Yamamoto and Ohtsuki, 1989; Goodin and Aminoff, 1992). Our study adds three important new pieces of information about this modification mechanism. First, we show reflex modulation in anticipation of altered interactions with the environment. In contrast, previous studies focused on reflex modulation according to the subject's intention to make a voluntary action (Evarts and Tanji, 1974, 1976; Marsden et al., 1976a). Second, we show that the modulation of reflex gains occurs within the sensorimotor cortex itself (see below, Cortical involvement in reflex gain modulation). Finally, we show that anticipatory reflexes make a functional contribution to reaction force, which probably reflects hand stiffness. This is consistent with the role of stretch reflexes in dynamic stiffness regulation (Lacquaniti and Maioli, 1989; Hore et al., 1990; Bennett et al., 1994; Gomi et al., 2002).

\section{Cortical involvement in reflex gain modulation}

Our central interest has been how such reflex gain modulation is coded in the brain. This study therefore attempted to clarify the role of interneuronal networks within M1 in reflex gain modulation using TMS. TMS over M1 results in long-lasting suppression of voluntary EMG activity (SP) after the MEP. Several studies have reported that the major part of this SP (>50 ms after TMS) arises from TMS-induced activation of networks of inhibitory interneurons projecting to pyramidal cells in $\mathrm{M} 1$ with $\mathrm{GABA}_{\mathrm{B}}$ mediated slow inhibitory connections (Brasil-Neto et al., 1995; Nakamura et al., 1997; Chen, 2004). Conversely, the early part of SP $(<50 \mathrm{~ms})$ may involve spinal factors such as recurrent inhibition by Renshaw cell activation and/or afterhyperpolarization and/or descending inhibitory volleys (Inghilleri et al., 1993; Ziemann et al., 1993). From this viewpoint, we investigated the effect of the TMS-induced SP on stretch reflex responses by applying TMS $50 \mathrm{~ms}$ before the perturbation such that both the perturbation itself and the initiation of induced reflex response were mostly contained within the presumed cortical portion of the SP. Moreover, the short interval between TMS and perturbation implies that the reflex responses could not be reprogrammed after TMS delivery, but rather reflected the anticipatory CNS strategy used to control the movement before TMS. In these circumstances, the observed reduction in reflex gain modulation may be attributed to the effects of TMS on interneuronal excitability in M1 during the reflex processing period.

However, before reaching this conclusion, we must be confident that TMS-induced reductions in reflex gain modulation are attributable to the effects of TMS on the cortical interneuronal network. As described above, one could expect that the effect of TMS on force field-dependent reflex modulation merely arose from the overall reduction in amplitude. A control experiment, however, showed that low reflex amplitudes induced by smaller perturbations were quite compatible with reflex gain modulation (Fig. 5). This means that the loss of reflex modulation is not an epiphenomenon attributable to reduced reflex amplitude after TMS.

Another major concern is the neural locus of the TMSinduced suppression of reflex modulation. There are at least three possible locations where the gain of transcortical reflexes might be modulated: the sensorimotor cortex itself, the spinal cord, and the peripheral fusimotor system (i.e., muscle spindle sensitivity) (Prochazka, 1989). As for the last possible location, the twitches of extrafusal muscle fibers produced by TMS could have interfered with fusimotor action so as to disrupt preset reflex gain. However, an additional control experiment showed that reflex gain modulation was not reduced when muscle twitches, similar to those caused by TMS, were induced by peripheral nerve stimulation that did not affect the cortex directly (Fig. 6). This suggests either that such muscle twitches have little effect on fusimotor operation, and/or that the fusimotor gain changes play little role in the reflex gain modulation observed in our studies.

Our experiments did not specifically investigate whether the 
gains of spinal synaptic mechanisms were remotely affected by TMS over M1. Descending volleys generated by TMS could, in principle, remotely affect the spinal cord. However, several studies (Inghilleri et al., 1993; Brasil-Neto et al., 1995) have demonstrated that the SP duration induced by brainstem stimulation is very short compared with that induced by TMS to the cortex [ $\sim 43$ vs $234 \mathrm{~ms}$ for Inghilleri et al. (1993)]. This suggests that the contribution of the spinal SP to reflex processing should be small relative to the contribution of the "cortical" SP in the situation studied here. The presence of reflex modulation when peripheral nerve stimulation produced putative spinal silent period (Fig. 6) also suggests a minor role of spinal synaptic mechanisms in the reflex modulation. This follows because the SP induced by mixed nerve stimulation is thought to arise from spinal mechanisms such as recurrent inhibition and/or tendon-organ activity concomitant muscle twitches (Taylor et al., 1999), although this stimulation may activate the spinal cord circuitry in a quite different way from the activation attributable to TMS.

On the basis of this combined evidence, we suggest that TMS over M1 acted to reduce reflex gain modulation at cortical rather than spinal synapses. A positron emission tomography and TMS study (Paus et al., 1997) pointed out that the excitation of remote brain regions by repetitive TMS reflected orthodromic effects of TMS, based on a comparison between orthodromic and antidromic effects on synaptic activity (Wong and Moss, 1992). Therefore, the elimination of reflex gain modulation by singlepulse TMS may have occurred predominantly, although perhaps not exclusively, within the sensorimotor cortex, and locally under the site of stimulation.

\section{Reflex generation and modulation}

A notable aspect in our results is that (1) the stretch reflex response appeared clearly even when the SP after TMS partially overlapped with the reflex response, (2) but its gain modulation was significantly reduced (Fig. 4C). This combination suggests that the neural mechanism mediating sensory signals (reflex generation mechanism) is partly independent of the mechanism modulating them (reflex modulation mechanism). Previous studies have shown that the occurrence of reflex responses at transcortical latencies cannot be voluntarily suppressed but that the amplitude of these responses is under voluntary control (Marsden et al., 1976a; Yamamoto and Ohtsuki, 1989). If the excessive inhibition in interneuronal circuits induced by TMS had simply influenced reflex amplitude (generation), then reflex gain modulation would be expected to remain while its amplitude decreased, like the results of control experiments using small perturbations (Fig. 5) and using peripheral nerve stimulation (Fig. 6). However, our main study rather found the opposite effect: disappearance of reflex gain modulation despite continued generation of a reflex response. This suggests that the SP after TMS selectively influences reflex modulation rather than reflex generation. One explanation for the dissociable effects of SP on reflex generation and reflex modulation might be the existence of two parallel, direct and interneuronal routes in the cortex between sensory and motor cortices. For instance, some neurons in the primary sensory cortex terminate directly on pyramidal neurons in M1 (Zarzecki et al., 1978; Ghosh and Porter, 1988). The generation of a reflex per se might reflect the direct route. Reflex modulation might involve an additional, indirect route. This would be mediated by interneuronal networks within $\mathrm{M} 1$, and would be affected by the SP. From a functional viewpoint, we suggest that these interneuronal networks may preparatorily adjusting reflex gains according to task demands. This modulation of reflexes may be basically similar to the known modification of such circuits during voluntary movements (Mathis et al., 1998, 1999; Hess et al., 1999; Tinazzi et al., 2003).

\section{References}

Bawa P, Hamm JD, Dhillon P, Gross PA (2004) Bilateral responses of upper limb muscles to transcranial magnetic stimulation in human subjects. Exp Brain Res 158:385-390.

Bennett DJ, Gorassini M, Prochazka A (1994) Catching a ball: contributions of intrinsic muscle stiffness, reflexes, and higher other responses. Can J Physiol Pharmacol 72:525-534.

Bonnet M (1983) Anticipatory changes of long-latency stretch responses during preparation for directional hand movements. Brain Res 280:51-62.

Brasil-Neto JP, Cammarota J, Valls-Solé J, Pascual-Leone A, Hallett M, Cohen LG (1995) Role of intracortical mechanisms in the late part of the silent period to transcranial stimulation of the human motor cortex. Acta Neurol Scand 92:383-386.

Burdet E, Osu R, Franklin DW, Yoshioka T, Milner TE, Kawato M (2001) The central nervous system stabilizes unstable dynamics by learning optimal impedance. Nature 414:446-449.

Chen R (2004) Interactions between inhibitory and excitatory circuits in the human motor cortex. Exp Brain Res 154:1-10.

Colebatch JG, Gandevia SC, McCloskey DI, Potter EK (1979) Subject instruction and long latency reflex responses to muscle stretch. J Physiol (Lond) 292:527-534.

Conrad B, Dressler D, Benecke R (1984) Changes of somatosensory evoked potentials in man as correlates of transcortical reflex mediation. Neurosci Lett 46:97-102.

Day BL, Rothwell JC, Thompson PD, Maertens de Noordhout A, Nakashima K, Shannon K, Marsden CD (1989) Delay in the execution of voluntary movement by electrical or magnetic brain stimulation in intact man. Evidence for the storage of motor programs in the brain. Brain 112:649-663.

Day BL, Riescher H, Struppler A, Rothwell JC, Marsden CD (1991) Changes in the response to magnetic and electrical stimulation of the motor cortex following muscle stretch in man. J Physiol (Lond) 433:41-57.

Dietz V, Discher M, Trippel M (1994) Task-dependent modulation of short- and long-latency electromyographic responses in upper limb muscles. Electroencephalogr Clin Neurophysiol 93:49-56.

Evarts EV, Tanji J (1974) Gating of motor cortex reflexes by prior instruction. Brain Res 71:479-494.

Evarts EV, Tanji J (1976) Reflex and intended responses in motor cortex pyramidal tract neurons of monkey. J Neurophysiol 39:1069-1080.

Franklin DW, Burdet E, Osu R, Kawato M, Milner TE (2003) Functional significance of stiffness in adaptation of multijoint arm movements to stable and unstable dynamics. Exp Brain Res 151:145-157.

Ghosh S, Porter R (1988) Corticocortical synaptic influences on morphologically identified pyramidal neurones in the motor cortex of the monkey. J Physiol (Lond) 400:617-629.

Goodin DS, Aminoff MJ (1992) The basis and functional role of the late EMG activity in human forearm muscles following wrist displacement. Brain Res 589:39-47.

Gomi H, Kawato M (1996) Equilibrium-point control hypothethis examined by measured arm stiffness during multijopint movement. Science 272:117-120.

Gomi H, Kawato M (1997) Human arm stiffness and equilibrium-point trajectory during multi-joint movement. Biol Cybern 76:163-171.

Gomi H, Osu R (1998) Task-dependent viscoelasticity of human multijoint arm and its spatial characteristics for interaction with environments. J Neurosci 18:8965-8978.

Gomi H, Saijo N, Haggard P (2002) Coordination of multi-joint arm reflexes is modulated during interaction with environments. Paper presented at 12th Annual Meeting of the Society for the Neural Control of Movement, Naples, Florida, April.

Hallett M, Bielawski M, Marsden CD (1981) Behavior of the long-latency stretch reflex prior to voluntary movement. Brain Res 219:178-185.

Hess A, Kunesch E, Classen J, Hoeppner J, Stefan K, Benecke R (1999) Taskdependent modulation of inhibitory actions within the primary motor cortex. Exp Brain Res 124:321-330.

Hore J, McCloskey DI, Taylor JL (1990) Task-dependent changes in gain of 
the reflex response to imperceptible perturbations of joint position in man. J Physiol (Lond) 429:309-321.

Inghilleri M, Berardelli A, Cruccu G, Manfredi M (1993) Silent period evoked by transcranial stimulation of the human cortex and cervicomedullary junction. J Physiol (Lond) 466:521-534.

Johnson MTV, Kipnis AN, Lee MC, Ebner TJ (1993) Independent control of reflex and volitional EMG modulation during sinusoidal pursuit tracking in humans. Exp Brain Res 96:347-362.

Kimura T, Gomi H, Saijo N, Haggard P (2004) Transcranial magnetic stimulation of the motor cortex disrupts anticipatory setting of arm reflex gain according to dynamical interaction. Paper presented at 14th Annual Meeting of the Society for the Neural Control of Movement, Sitges, Spain, March 27-April 3.

Lacquaniti F, Maioli C (1989) The role of preparation in tuning anticipatory and reflex responses during catching. J Neurosci 9:134-148.

Lewis GN, Polych MA, Byblow WD (2004) Proposed cortical and subcortical contributions to the long-latency stretch reflex in the forearm. Exp Brain Res 156:72-79.

Marsden CD, Merton PA, Morton HB (1976a) Servo action in the human thumb. J Physiol (Lond) 257:1-44.

Marsden CD, Merton PA, Morton HB (1976b) Stretch reflex and servo action in a variety of human muscles. J Physiol (Lond) 259:531-560.

Mathis J, de Quervain D, Hess CW (1998) Dependence of the transcranially silent period on the "instruction set" and the individual reaction time. Electroencephalogr Clin Neurophysiol 109:426-435.

Mathis J, de Quervain D, Hess CW (1999) Task-dependent effects on motor-evoked potentials and on the following silent period. J Clin Neurophysiol 16:556-565.

Matthews PWC (1986) Observations on the automatic compensation of reflex gain on varying the pre-existing level of motor discharge in man. J Physiol (Lond) 390:73-90.

Matthews PWC (1991) The human stretch reflex and the motor cortex. Trends Neurosci 14:87-91.

Mussa-Ivaldi FA, Hogan N, Bizzi E (1985) Neural, mechanical, and geometric factors subserving arm posture in humans. J Neurosci 5:2732-2743.

Nakamura H, Kitagawa H, Kawauchi Y, Tsuji H (1997) Intracortical facili- tation and inhibition after transcranial magnetic stimulation in conscious human. J Physiol (Lond) 498:817-823.

Paus T, Jech R, Thompson CJ, Comeau Roch, Peters T, Evans AC (1997) Transcranial magnetic stimulation during positron emission tomography: new method for studying connectivity of the human cerebral cortex. J Neurosci 17:3178-3184.

Prochazka A (1989) Sensorimotor gain control: a basic strategy of motor systems? Prog Neurobiol 33:281-307.

Sullivan SJ, Hayes KC (1987) Changes in short and long latency stretch reflexes prior to movement initiation. Brain Res 412:139-143.

Taylor JL, Butler JE, Gandevia SC (1999) Altered responses of human elbow flexors to peripheral-nerve and cortical stimulation during a sustained maximal voluntary contraction. Exp Brain Res 127:108-115.

Tinazzi M, Farina S, Tamburin S, Facchini S, Fiaschi A, Restivo D, Berardelli A (2003) Task-dependent modulation of excitatory and inhibitory functions within the human primary motor cortex. Exp Brain Res 150:222-229.

Triggs WJ, Cros D, Macdonell RAL, Chiappa KH, Fang J, Day BJ (1993) Cortical and spinal motor excitability during the transcranial magnetic stimulation silent period in humans. Brain Res 628:39-48.

Wang T, Dordevic GS, Shadmehr R (2001) Learning the dynamics of reaching movements results in the modification of arm impedance and longlatency perturbation responses. Biol Cybern 85:437-448.

Wong M, Moss RL (1992) Modulation of single-unit activity in the rat amygdala by neurotransmitters, estrogen priming, and synaptic inputs from the hypothalamus and midbrain. Synapse 10:94-120.

Yamamoto C, Ohtsuki T (1989) Modulation of stretch reflex by anticipation of the stimulus through visual information. Exp Brain Res 77:12-22.

Zarzecki P, Shinoda Y, Asanuma H (1978) Projections from area 3a to the motor cortex by neurons activated from group I muscle afferents. Exp Brain Res 33:269-282.

Ziemann U, Netz J, Szelényi A, Hömberg V (1993) Spinal and supraspinal mechanisms contribute to the silent period in the contracting soleus muscle after transcranial magnetic stimulation of human motor cortex. Neurosci Lett 156:167-171. 